Pacific Journal of Mathematics

SOME PROPERTIES OF SEQUENCES, WITH AN
APPLICATION TO NONCONTINUABLE POWER SERIES 


\section{SOME PROPERTIES OF SEQUENCES, WITH AN APPLICATION TO NONCONTINUABLE POWER SERIES}

F. W. Carroll

For a real sequence $f=\{f(n)\}$ and positive integer $N$, let $F^{N}$ denote the sequence of $N$-tuples $\{(f(n+1), \cdots, f(n+N))\}$. A functional equation method due to Kemperman is used to obtain a sufficient condition on $s$ in order that $s^{N}$ have an independent $N$-tuple among its cluster points. If a bounded $s$ has the latter property, and if $g=r s$, where $r(n) \rightarrow \infty$ and $r(n+1) / r(n) \rightarrow 1$ as $n \rightarrow \infty$, then there is a subsequence $S$ of the sequence of positive integers such that, for almost all real $\alpha$, the restriction of $\alpha g^{N}$ to $S$ is uniformly distributed $(\bmod 1)$ in the $N$-cube.

Let $F$ be an analytic function whose Maclaurin series has bounded coefficients $\left\{a_{n}\right\}$ which satisfy the additional requirement

$$
\lim _{M \rightarrow \infty} \inf _{0 \leqq k<\infty} \sum_{n=k}^{k+M}\left|a_{n}\right|=\infty \text {. }
$$

If $a_{n}=\left|a_{n}\right| \exp \{2 \pi i f(n)\}$, then the density $(\bmod 1)$ of $f^{N}$ for each $N$ is sufficient in order that $F$ have the unit circle as a natural boundary. Hence, a metric result for noncontinuable series is obtained from the results for sequences.

1. Notation. For $x$ real, let $((x))=x-[x]$, and $e(x)=\exp (2 \pi i x)$. $h_{1}, \cdots, h_{N^{r}}$ will denote an $N$-tuple of integers, not all of which are zero. The sequence of nonnegative integers will be denoted by $Z$, and subsequences of $Z$ by $S_{1}, S_{2}$, etc. For a real sequence $f$, we denote by $\Delta f$ the sequence $\{f(n+1)-f(n)\}$ and

$$
\Delta^{j+1} f=\Delta\left(\Delta^{j} f\right),(j=1,2, \cdots)
$$

\section{The property (PN).}

Definition. A bounded sequence $s$ of real numbers will be said to have property $(\mathrm{PN})$ if there is an independent $N$-tuple among the cluster points of $s^{N}$. In other words, $s$ has property (PN) if there is a subsequence $S$ of $Z$ such that for every $N$-tuple $h_{1}, \cdots, h_{N}$ of integers not all zero, there holds

$$
\lim _{n \rightarrow \infty}\left|h_{1} s(n+1)+\cdots+h_{N} s(n+N)\right|>0, \quad(n \in S) .
$$

We shall be interested in sequences $s$ of the following form: 


$$
s(n)=\varphi(\psi(n)),
$$

where $\varphi$ is a function of period 1 with at most a nowhere dense set of points of discontinuity, and $\psi$ has the property (QN).

(QN) There exists a subsequence $S_{1}$ of $Z$ such that

(i) $\Delta^{j} \psi(n)$ converges $(\bmod 1)$ for $n \rightarrow \infty$,

$$
n \in S_{1} \quad(j=2, \cdots, N)
$$

(ii) $\left\{\left(\left((\psi(n)),((\Delta \psi(n))): n \in S_{1}\right\}\right.\right.$ is not nowhere dense.

THEOREM 2.1. Let $s$ be of the form (2.2), where $\phi$ and $\psi$ have the properties listed above. Then either $s$ has property (PN), or else $\varphi$ agrees on some interval $I \subset[0,1]$ with a polynomial of degree $N$ 2 at most.

Proof. Under the conditions on $\varphi$ and $\psi$, it is possible to obtain a subsequence $S_{2}$ of $S_{1}$ and an open disk $D$ in the plane such that

$$
\begin{aligned}
& \text { (i) } \lim _{n \rightarrow \infty} \Delta^{j} \psi(n)=\tau_{j} \quad(\bmod 1), \quad\left(n \in S_{2}\right), \quad(j=2, \cdots, N), \\
& \text { (ii) }\left\{\left(\left((\psi(n)),((\Delta \psi(n))): n \in S_{2}\right\} \text { is dense in } D,\right.\right. \\
& \text { (iii) for every }\left(\tau_{0}, \tau_{1}\right) \text { in } D \text {, and } \\
& \text { every } p, 1 \leqq p \leqq N, \text { the point } \\
& \qquad \tau_{0}+p \tau_{1}+\sum_{j=2}^{p}\left(\begin{array}{c}
p \\
j
\end{array}\right) \tau_{j}
\end{aligned}
$$

is a point of continuity for $\varphi$.

For each $\left(\tau_{0}, \tau_{1}\right)$ in $D$, a subsequence $S_{3}=S_{3}\left(\tau_{0}, \tau_{1}\right)$ of $S_{2}$ can be chosen so that the corresponding subsequence of (2.4 (ii)) converges to $\left(\tau_{0}, \tau_{1}\right)$. In this case, as $n \rightarrow \infty, n \in S_{3}$, one has for every $h_{1}, \cdots, h_{N}$,

$$
\begin{aligned}
\lim _{n \rightarrow \infty} \sum_{p=1}^{N} h_{p} s(n+p)= & \lim _{n \rightarrow \infty} \sum_{p=1}^{N} h_{p} \varphi(\psi(n) \\
& \left.+p \Delta \psi(n)+\sum_{j=2}^{p}\left(\begin{array}{c}
p \\
j
\end{array}\right) \Delta^{j} \psi(n)\right)
\end{aligned}
$$

so that

$$
\lim _{n \rightarrow \infty} \sum_{p=1}^{N} h_{p} s(n+p)=\sum_{p=1}^{N} h_{p} \varphi\left(\tau_{0}+p \tau_{1}+\sum_{j=2}^{p}\left(\begin{array}{c}
p \\
j
\end{array}\right) \tau_{j}\right), \quad\left(n \in S_{3}\right) .
$$

Suppose now that $s$ does not have property (PN). Then for each $\left(\tau_{0}, \tau_{1}\right)$ in $D$, there is an $N$-tuple $h_{1}, \cdots, h_{N}$ such that the right hand member of (2.5) is zero. Hence $D$ is a countable union of closed sets

$$
F=F\left(h_{1}, \cdots, h_{N}\right)=\left\{\left(\tau_{0}, \tau_{1}\right) \in D:(2.5) \text { vanishes }\right\} .
$$

Some $F$, then, must contain an open subdisk $D_{1}$, with center 
$\left(\tau_{0}^{\prime}, \tau_{1}^{\prime}\right)$. That is, there exists an $N$-tuple $h_{1}, \cdots, h_{N}$ of integers not all zero with the property that for all sufficiently small positive $h$ and $k$,

$$
\sum_{p=1}^{N} h_{p} \varphi\left(h+p k+\tau_{0}^{\prime}+p \tau_{1}^{\prime}+\sum_{j=2}^{p}\left(\begin{array}{c}
p \\
j
\end{array}\right) \tau_{j}\right)=0 .
$$

The assertion of the theorem follows upon taking

$$
\varphi_{p}(x)=h_{p} \varphi\left(x+\tau_{0}^{\prime}+p \tau_{1}^{\prime}+\sum_{j=2}^{p}\left(\begin{array}{c}
p \\
j
\end{array}\right) \tau_{j}\right)
$$

in the following lemma, a weak version of one due to Kemperman [4, p. 41]. The proof is included for completeness.

LEMma. Let $a>0$, and let $\varphi_{1}, \cdots, \varphi_{N}$ be real functions, with $\varphi_{j}$ defined and continuous on $I_{j}=(-(j+1) a,(j+1) a),(j=1, \cdots, N)$. Suppose that for all $x, y$ in $(-a, a)$, there holds

$$
\sum_{j=1}^{N} \varphi_{j}(x+j y)=0 \text {. }
$$

Then $\varphi_{j}$ is equal on $I_{j}$ to a polynomial of degree $N-2$ at most.

Proof. We may suppose that $N \geqq 2$ (the case $N=1$ is trivial), and that the lemma holds for $N-1$. Let $0<b<a$, and let $I_{j}^{\prime}=$ $(-(j+1) b,(j+1) b)$.

Next, we choose and keep fixed a number $h, 0<h<\min (b, a-b)$. For this $h$, and $j=1, \cdots, N$, let

$$
\widetilde{\varphi}_{j}(x)=\varphi_{j}(x+(1-j / N) h)-\varphi_{j}(x), \quad\left(x \in I_{j}^{\prime}\right) .
$$

We note that each $\widetilde{\varphi}_{j}$ is continuous, and $\widetilde{\varphi}_{N} \equiv 0$. Moreover, if $x, y$ are in $(-b, b)$, then $x, y, x+h$, and $y-h / N$ are in $(-a, a)$. Thus, for all $x, y$ in $(-b, b)$, we have

$$
\sum_{j=1}^{N-1} \widetilde{\varphi}_{j}(x+j y)=\sum_{j=1}^{N} \varphi_{j}(x+h+j(y-h / N))-\sum_{j=1}^{N} \varphi_{j}(x+j y)=0 .
$$

The induction hypothesis implies that, for $j=1, \cdots, N-1, \widetilde{\varphi}_{j}$ is a polynomial of degree $N-3$ at most on $I_{j}^{\prime}$. Hence $\varphi_{j}$ is, on $I_{j}^{\prime}$, the sum of a polynomial of degree $N-2$ at most and a function of period $(1-j / N) h$. But such a representation is given for every sufficiently small positive $h$, which, with the continuity of $\varphi_{j}$, implies that $\varphi_{j}$ is a polynomial of degree $N-2$ at most on $I_{j}^{\prime},(1 \leqq j \leqq N-1)$. From the arbitrariness of $b, \varphi_{j}$ is such a polynomial on $I_{j}$. Finally, (2.6) shows that $\varphi_{N}$ is also such a polynomial on $I_{N}$.

In a previous paper [1], results of $v$. $d$. Corput were used to 
obtain various sufficient conditions on a real sequence $\psi$ in order that $\psi$ satisfy condition (I):

(I) There exists a sequence $S$ such that $\lim \Delta^{j} \psi(n)(n \in S)$ exists for all $j \geqq r$, while $\left\{\left(\psi(n), \Delta \psi(n), \cdots, \Delta^{r-1} \psi(n)\right): n \in S\right\}$ is uniformly distributed $(\bmod 1)$ in the r-dimensional unit cube.

(I) clearly implies that $\psi$ has property (QN) for every $N \geqq 2$. The reader is referred to the paper for details and proofs.

\section{A metric result for uniform distribution in the $N$-cube.}

THEOREM 3.1 Let $g=\{g(n): n \in Z\}$ be a sequence of real numbers. Let there exist a subsequence $S_{5}$ of $Z$ such that, for every $N$-tuple $h_{1}, \cdots, h_{N}$ of integers not all zero there holds

$$
\lim \left|\sum_{p=1}^{N} h_{p} g(n+p)\right|=\infty, \quad \text { as } n \rightarrow \infty, n \in S_{0} .
$$

Then there exists a subsequence $S$ of $S_{0}$ such that, for almost all real $\alpha$, the sequence $\left(\alpha g^{N}\right) \mid S$ is uniformly distributed $(\bmod 1)$ in the $N$ cube.

Proof. Let the set of all such $N$-tuples be ordered, with, say, $h_{1}^{\prime}, \cdots, h_{N}^{\prime}$ as the first. Let a subsequence $S_{1} \subset S_{0}$ be taken such that

$$
\sum_{p=1}^{N} h_{p}^{\prime}\{g(n+p)-g(m+p)\}
$$

is either greater than 1 for every $n, m$ in $S_{1}$, with $n>m$, or else is less than -1 for every such $n$ and $m$. Successively extracting subsequences $S_{1} \supset S_{2} \supset \cdots$ in this way, and then using a diagonal procedure, one finally obtains a sequence $S$ such that, for every $N$-tuple $h_{1}, \cdots, h_{N}$, there is an $m_{0}=m_{0}\left(h_{1}, \cdots, h_{N}\right)$ such that one has either

$$
\sum_{p=1}^{N} h_{p}\{g(n+p)-g(m+p)\} \geqq 1
$$

for all $n$ and $m$ in $S$ with $n>m \geqq m_{0}$ or else

$$
\sum_{p=1}^{N} h_{p}\{g(n+p)-g(m+p)\} \leqq-1
$$

for all such $n$ and $m$.

By a well-known result of Weyl [6, p. 348], either condition (3.2) or (3.3) implies that, for almost all real $\alpha$, the sequence

$$
\alpha \sum_{p=1}^{N} h_{p} g(n+p)
$$


is uniformly distributed $(\bmod 1)$. There being only countably many $N$-tuples, it follows that, for almost all $\alpha$, (3.4) is uniformly distributed $(\bmod 1)$ for every $N$-tuple $h_{1}, \cdots, h_{N}$. But this shows [2, p. 66] that for almost all $\alpha$ the sequence $\left(\alpha g^{N}\right) \mid S$ is uniformly distributed $(\bmod 1)$ in the $N$-cube.

It is easy to see that if $\theta>1$ is a transcendental number and $g(n)=\theta^{n}$, then Theorem 3.1 is applicable. The next result shows the less obvious fact that Theorem 3.1 also applies if, for instance, $g(n)=$ $n^{3} \log n \sin n^{2}$.

THEOREM 3.2. Let $g=\{g(n): n \in z\}$ be of the form

$$
g(n)=r(n) s(n),
$$

$n \in Z$,

where s has property (PN), while

$$
\lim r(n)=\infty, \quad \lim (r(n+1) / r(n))=1 .
$$

Then there is a subsequence $S_{0}$ of $Z$ such that (3.1) holds for every $N$-tuple $h_{1}, \cdots, h_{N}$ of integers not all zero.

Proof. For $p=1,2, \cdots, N$, it follows from (3.6) that

$$
r(n+p)=r(n)(1+0(1)), \quad \text { as } n \rightarrow \infty .
$$

Therefore we have

$$
g(n+p)=r(n) s(n+p)(1+o(1)), \quad \text { as } n \rightarrow \infty, \quad p=1, \cdots, N
$$

Since $s$ has property (PN), there exists a subsequence $S_{0}$ of $Z$ such that

$$
\lim _{n \rightarrow \infty}\left|h_{1} s(n+1)+\cdots+h_{N} s(n+N)\right|>0,
$$

for all $N$-tuples $h_{1}, \cdots, h_{N}$ of integers not all zero. But (3.6), (3.7), and (2.1) imply (3.1).

4. An application to noncontinuable power series, Perry [5] has proved that, for every real sequence $f=\{f(n): n \in Z\}$, there exists a sequence of moduli $\left\{\left|a_{n}\right|: n \in Z\right\}$ such that the power series

$$
\sum_{n=0}^{\infty}\left|a_{N}\right| e(f(n)) z^{n}
$$

has radius of convergence 1 and the analytic function it represents can be continued analytically across a semicircle of the unit circle. However, if the additional requirements

$$
\left|a_{n}\right|=0(1)
$$


and

$$
\lim _{N \rightarrow \infty} \inf _{0 \leqq k<\infty} \sum_{n=k+1}^{k+N}\left|a_{n}\right|=\infty
$$

are imposed, then there are conditions on $f$ sufficient that (4.1) represent a function with $|z|=1$ as its natural boundary. Some such conditions were given in [1]. Theorem 4 gives a metric result in this direction.

THEorem 4. Let $\left\{\left|a_{n}\right|: n \in Z\right\}$ satisfy (4.2) and (4.3). Let $g$ be a real sequence which, for each $N$, satifies the hypothesis of Theorem 3.1. For each real $\alpha$, let

$$
F_{\alpha}(z)=\sum_{n=0}^{\infty}\left|a_{n}\right| e(\alpha g(n)) z^{n}
$$$$
|z|<1 \text {. }
$$

Then the set of $\alpha$ for which $F_{\alpha}$ can be continued across an arc of the unit circle has measure zero.

Example. $\sum e\left(\alpha n \sin n^{2}\right) z^{n}$ has $|z|=1$ as its natural boundary for almost all $a$.

For $N=2,3, \cdots$, let $A_{N}$ be the set of those real $\alpha$ for which $\alpha g^{N}$ is dense $(\bmod 1)$ in the unit $N$-cube.

By Theorem 3.1, $A_{N}$ contains almost all $\alpha$, and it follows that almost all $\alpha$ are in $A_{N}$ for every $N$. For each such $\alpha$, and each $z_{0}=e\left(\theta_{0}\right)$, there holds

$$
\limsup _{k \rightarrow \infty}\left|\sum_{k+1}^{k+N} a_{n} e\left(\alpha g(n)+n \theta_{0}\right)\right| \geqq \liminf _{k \rightarrow \infty} \sum_{k+1}^{k+N}\left|a_{n}\right| .
$$

In view of (4.3), (4.5) shows that the partial sums of the series in (4.4) are unbounded at $z_{0}$. By (4.2) and a well-known theorem of Fatou [3, p. 391], it follows that $z_{0}$ is a singularity for $F$.

\section{REFERENCES}

1. F. W. Carroll, On some classes of noncontinuable analytic functions, Trans. Amer. Math. Soc. 94 (1960), 74-85.

2. J. W. Cassells, An introduction to Diophantine approximation, Cambridge Tract 45, Cambridge Univ. Press, 1957.

3. P. Fatou, Séries trigonométriques et séries de Taylor, Acta Math. 30 (1906), 335-400. 4. J.H.B. Kemperman, A general functional equation, Trans. Amer. Math. Soc. 86 (1957), 28-56.

5. R. L. Perry, A theorem on power series whose coefficients have given arguments, J. Lond. Math. Soc. 35 (1960), 172-176.

6. H. Weyl, Gleichverteilung von Zahlen mod Eins, Math. Ann. 77 (1916), 313-352.

Received September 19, 1966. This research was supported by a grant from the National Science Foundation.

The OHio State University

Columbus, OHio 


\section{PACIFIC JOURNAL OF MATHEMATICS}

\section{EDITORS}

\section{H. ROYDEN}

Stanford University

Stanford, California

\section{J. P. Jans}

University of Washington

Seattle, Washington 98105

\section{J. DUGUNDJI}

Department of Mathematics

Rice University

Houston, Texas 77001

RICHARD ARENS

University of California

Los Angeles, California 90024

\section{ASSOCIATE EDITORS}
E. F. BeCKenbaCH
B. H. NeumanN
F. WOLF
K. YosIDA

\section{SUPPORTING INSTITUTIONS}

UNIVERSITY OF BRITISH COLUMBIA CALIFORNIA INSTITUTE OF TECHNOLOGY UNIVERSITY OF CALIFORNIA MONTANA STATE UNIVERSITY UNIVERSITY OF NEVADA NEW MEXICO STATE UNIVERSITY OREGON STATE UNIVERSITY UNIVERSITY OF OREGON OSAKA UNIVERSITY UNIVERSITY OF SOUTHERN CALIFORNIA
STANFORD UNIVERSITY UNIVERSITY OF TOKYO UNIVERSITY OF UTAH WASHINGTON STATE UNIVERSITY UNIVERSITY OF WASHINGTON

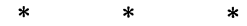

AMERICAN MATHEMATICAL SOCIETY CHEVRON RESEARCH CORPORATION TRW SYSTEMS

NAVAL ORDNANCE TEST STATION 


\section{Pacific Journal of Mathematics}

\section{Vol. 24, No. $1 \quad$ May, 1968}

Harry P. Allen, Lie algebras of type $D_{4}$ over algebraic number fields ...... 1

Charles Ballantine, Products of positive definite matrices. II............ 7

David W. Boyd, The spectral radius of averaging operators ............ 19

William Howard Caldwell, Hypercyclic rings ................... 29

Francis William Carroll, Some properties of sequences, with an application

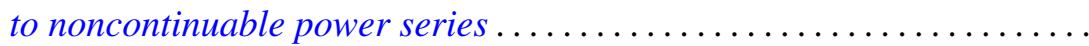

David Fleming Dawson, Matrix summability over certain classes of

sequences ordered with respect to rate of convergence ........... 51

D. W. Dubois, Second note on David Harrison's theory of preprimes. . . . . 57

Edgar Earle Enochs, A note on quasi-Frobenius rings.............. 69

Ronald J. Ensey, Isomorphism invariants for Abelian groups modulo bounded groups ................................ 71

Ronald Owen Fulp, Generalized semigroup kernels ................ 93

Bernard Robert Kripke and Richard Bruce Holmes, Interposition and approximation ................................. 103

Jack W. Macki and James Sai-Wing Wong, Oscillation of solutions to second-order nonlinear differential equations ..................

Lothrop Mittenthal, Operator valued analytic functions and generalizations

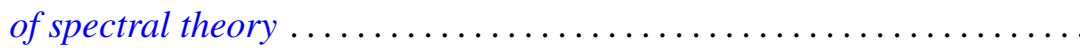

T. S. Motzkin and J. L. Walsh, A persistent local maximum of the pth power deviation on an interval, $p<1 \ldots \ldots \ldots \ldots \ldots \ldots \ldots \ldots \ldots \ldots . \ldots \ldots$

Jerome L. Paul, Sequences of homeomorphisms which converge to homeomorphisms ...........................

Maxwell Alexander Rosenlicht, Liouville's theorem on functions with elementary integrals.

Joseph Goeffrey Rosenstein, Initial segments of degrees .

$\mathrm{H}$. Subramanian, Ideal neighbourhoods in a ring ............

Dalton Tarwater, Galois cohomology of abelian groups . .

James Patrick Williams, Schwarz norms for operators ... .

Raymond Y. T. Wong, A wild Cantor set in the Hilbert cube. 